\title{
Isolantes térmicos produzidos a partir de resíduos sólidos industriais
}

\section{(Thermal insulators produced from industrial solid wastes)}

\author{
S. Arcaro $^{1,3}$, A. Albertin $^{3,4}$, F. R. Cesconeto ${ }^{1,3}$, B. G. de Oliveira Maia ${ }^{1,3}$, \\ C. Siligardi ${ }^{4}, A$. P. Novaes de Oliveira ${ }^{1-3}$ \\ ${ }^{1}$ Programa de Pós-Graduação em Ciência e Engenharia de Materiais (PGMAT) \\ ${ }^{2}$ Departamento de Engenharia Mecânica (EMC) \\ ${ }^{3}$ Laboratório de Materiais Vitrocerâmicos (VITROCER) \\ Universidade Federal de Santa Catarina (UFSC), Campus Universitário - Trindade, Florianópolis, SC 88040-900 \\ ${ }^{4}$ Università degli Studi di Modena e Reggio Emilia (UNIMORE), Modena, MO, Itália \\ *bianca.maia@outlook.com
}

\begin{abstract}
Resumo
Composições contendo dois diferentes tipos de resíduos, vidro de garrafas transparentes, VGT (50-70\%), e lodo de estação de tratamento de efluentes, ETE (30-50\%), e formador de poros, $\mathrm{CaCO}_{3}(2-10 \%)$, foram preparadas para a produção de isolantes térmicos para fins estruturais. As matérias-primas das composições formuladas foram, em uma etapa posterior, misturadas, compactadas uniaxialmente a $40 \mathrm{MPa}$ e, após secagem $\left(110{ }^{\circ} \mathrm{C} / 2 \mathrm{~h}\right.$ ), queimadas entre 750 e $1000{ }^{\circ} \mathrm{C}$ com patamares de 15 a 60 min. As matérias-primas e os materiais obtidos foram caracterizados do ponto de vista de suas propriedades químicas, físicas e microestruturais. Os resultados obtidos mostraram que é possível produzir isolantes térmicos, a partir de composições otimizadas contendo $70 \%$ de VGT, $30 \%$ de lodo de ETE e $5 \%$ de $\mathrm{CaCO}_{3}$, queimados entre 750 e $1000{ }^{\circ} \mathrm{C} / 60 \mathrm{~min}$, os quais apresentaram porosidades entre 3 e $62 \%$ com condutividades térmicas entre 1,25 e $0,5 \mathrm{~W} / \mathrm{m}$.K e com resistência à compressão entre 8,7 e 5,4 MPa. Os materiais obtidos são fortes candidatos em aplicações estruturais tais como painéis para isolamento térmico, os quais requerem uma combinação adequada de condutividade térmica, porosidade e resistência mecânica.

Palavras-chave: materiais porosos, resíduos sólidos, vidros, cerâmicas.
\end{abstract}

\begin{abstract}
In this work compositions containing two different wastes, transparent glass bottles, TGB (50-70\%), and sludge from sewage treatment plants, STP (30-50\%), and pore forming, $\mathrm{CaCO}_{3}(2-10 \%)$, were prepared for the production of thermal insulators. The mixed raw materials of the formulated compositions were uniaxially pressed (40 MPa) and the powder compacts were dried (110 ${ }^{\circ} \mathrm{C} / 2 \mathrm{~h}$ ) and fired in the temperature range between 750 and $1000{ }^{\circ} \mathrm{C}$ with holding times from 15 to 60 min. The raw materials and obtained materials were characterized in terms of their chemical, physical and microstructural properties. The results showed that it is possible to produce thermal insulators from optimized compositions containing $70 \% \mathrm{TGB}, 30 \% \mathrm{STP}$ and $5 \% \mathrm{CaCO}$ and fired between 750 and $1000{ }^{\circ} \mathrm{C}$ for $60 \mathrm{~min}$. The fired materials showed porosities between 3 and $62 \%$ with thermal conductivities between 1.25 and $0.5 \mathrm{~W} / \mathrm{m} . \mathrm{K}$ and compressive strength ranging from 8.7 to $5.4 \mathrm{MPa}$. On the basis of the obtained results it can be said, in general terms, that obtained porous materials are strong candidates for structural applications such as panels for thermal insulation which require an adequate combination of thermal conductivity, porosity and mechanical strength.

Keywords: porous materials, solid waste, glass, ceramics.
\end{abstract}

\section{INTRODUÇÃO}

Isolantes térmicos são materiais que possuem baixa condutividade térmica. Quando as temperaturas de serviço são maiores que a ambiente, os materiais tipicamente empregados são os cerâmicos. Neste caso, dependendo da composição química e da estrutura de poros do material, a condutividade térmica e a refratariedade podem variar significativamente, possibilitando assim a seleção de materiais com propriedades adequadas para aplicações específicas. O melhor isolamento térmico é o vácuo. Todavia, devido às dificuldades para obtê-lo e mantê-lo, sua aplicação é limitada. A solução prática é a utilização de ar que também apresenta uma baixa condutividade térmica $(0,026 \mathrm{~W} / \mathrm{mK})$ a $27^{\circ} \mathrm{C}$ [1]. Neste caso e considerando a convecção e a umidade presentes em sistemas com ar confinado, os quais aumentam a condutividade térmica, uma solução técnica é a utilização de materiais porosos ou celulares com poros fechados, tal que o ar ou outros gases da decomposição de agentes espumantes ou formadores de poros permaneçam enclausurados e estanques no interior dos poros formados. As cerâmicas porosas, incluindo-se os vidros, são materiais relativamente frágeis de elevada porosidade que apresentam estrutura celular com poros fechados, abertos ou interconectados [2]. O crescente interesse pelas cerâmicas porosas tem sido associado principalmente às suas propriedades específicas 
como elevada área superficial, elevada permeabilidade (caso das cerâmicas com poros abertos), baixas densidade e condutividade térmica, as quais estão ainda relacionadas com características próprias dos materiais cerâmicos, como elevadas refratariedade e resistência a ataques químicos. De fato, por causa destas propriedades, estas cerâmicas encontram aplicações tecnológicas, tais como filtros, isolantes térmicos, membranas, sensores de gás, suportes catalíticos, material estrutural leve, materiais para implantes ósseos, entre outras [3, 4]. Entre os materiais normalmente utilizados para a fabricação de materiais porosos, podese citar alumina, mulita, carbeto de silício, zircônia parcialmente estabilizada, hidroxiapatita e alguns sistemas compósitos, tais como carbeto de silício-alumina, aluminazircônia, alumina-mulita, mulita-zircônia [5-7]. Além disso, existe a possibilidade de se obter materiais porosos a partir de materiais vítreos ou parcialmente cristalinos, tais como vidros e vitrocerâmicas. Nestes casos, o uso de resíduos sólidos provenientes de indústrias cerâmicas é uma possibilidade explorada e que pode ser intensificada em aplicações envolvendo temperaturas menores que $500{ }^{\circ} \mathrm{C}$, como no caso de isolamentos de lareiras e churrasqueiras e sobretudo como painéis para isolamento térmico, não inflamável, e acústico de sistemas construtivos.

Os resíduos de vidros provenientes da indústria de embalagens utilizadas no cotidiano são descartados juntamente com o lixo doméstico [8]. Estes resíduos constituem cerca de $2 \%$ do total do lixo doméstico da cidade de São Paulo, o que equivale a um descarte de aproximadamente 7.000 t/mês [8]. Além disso, reciclar e utilizar esses materiais poderá contribuir com a matriz energética nacional através da economia de grande quantidade de energia, já que para produzir $1 \mathrm{~kg}$ de vidro novo são necessários $4500 \mathrm{~kJ}$, enquanto que para produzir $1 \mathrm{~kg}$ de vidro reciclado necessita-se de $500 \mathrm{~kJ}$ [8]. As indústrias de revestimentos cerâmicos também geram quantidades significativas de resíduos nas várias etapas do processo que nem sempre têm um destino adequado. Contudo, as maiores quantidades são geradas nos setores de preparação de esmaltes e tintas, preparação de massa e na linha de esmaltação/decoração [9]. De fato, em uma unidade fabril (a título de exemplo) que produz aproximadamente $300.000 \mathrm{~m}^{2} /$ mês de revestimentos cerâmicos, são gerados aproximadamente $192 \mathrm{~m}^{3}$ de efluentes líquidos, sendo que deste volume $117 \mathrm{~m}^{3}$ são provenientes dos setores de preparação de massa e de esmaltes e tintas e $75 \mathrm{~m}^{3}$ são oriundos da linha de esmaltação e de outros setores [9]. Todo este efluente líquido, após o processo de filtro-prensagem, gera enormes quantidades de resíduos, isto é, cerca de 30 t/mês [9]. O lodo cerâmico é principalmente constituído de sílica, alumina e alguns metais pesados e, por esta razão, sendo classificado como resíduo perigoso segundo a Norma ABNT-NBR 10.004 [10].

Desta forma, a reciclagem do resíduo pode desempenhar um importante papel na economia de matérias-primas naturais e na diminuição da poluição ambiental $[11,12]$. Isso pode ser conseguido por meio da substituição de uma ou mais matérias-primas da composição original por lodo cerâmico, respeitando-se as características tecnológicas do processo e as propriedades do produto [13]. Neste sentido, tem-se buscado diversas alternativas de reaproveitamento do lodo cerâmico, como na fabricação de produtos de cerâmica vermelha (tijolos e telhas) [14], na produção de esmaltes de baixa densidade para aplicação a disco em placas de revestimentos e na produção de fritas para a composição de engobes e esmaltes [15]. Desta maneira, pode-se alongar os ciclos de vida dos elementos, reduzindo as necessidades de sua extração do meio ambiente [16]. Isso é tanto mais necessário quando se considera que resíduos são, frequentemente, fontes de grandes problemas ambientais, mas poderiam ser soluções [16]. Neste contexto, este artigo reporta resultados de trabalho de pesquisa cujo objetivo principal foi a produção de materiais porosos processados a partir de vidros de garrafas descartadas e lodo da estação de tratamento de efluentes (ETE) de indústria de cerâmica de revestimento para isolamento térmico em sistemas construtivos que requerem uma relação adequada de condutividade térmica, porosidade e resistência mecânica.

\section{MATERIAIS E MÉTODOS}

Neste trabalho foram utilizados, como matériasprimas, vidro de garrafa transparente (VGT), lodo de ETE e carbonato de cálcio $\left(\mathrm{d}_{50}=2 \mu \mathrm{m}\right), \mathrm{CaCO}_{3}$ (Vetec Química Fina) como agente formador de poros. A composição química do VGT e do lodo de ETE, obtida por espectroscopia de fluorescência de raios X, FRX (Philips, PW 2400), é apresentada na Tabela I. O lodo de ETE (como recebido) empregado neste trabalho $\left(\mathrm{d}_{50}=10\right.$ $\mu \mathrm{m}$, determinado com granulômetro por difração a laser, Malvern Mastersizer 2000) foi cedido por uma empresa de cerâmica de revestimento da região.

O lodo cerâmico é principalmente constituído de sílica, alumina e alguns metais pesados e, por esta razão,

Tabela I - Composição química do VGT e do lodo de ETE. [Table I - Chemical composition of TGB and STP sludge].

\begin{tabular}{ccccccccccccc}
\hline \multicolumn{1}{c}{ Materiais } & \multicolumn{11}{c}{ Óxidos constituintes (\% em massa) } \\
\hline & $\mathrm{SiO}_{2}$ & $\mathrm{Al}_{2} \mathrm{O}_{3}$ & $\mathrm{Fe}_{2} \mathrm{O}_{3}$ & $\mathrm{CaO}$ & $\mathrm{K}_{2} \mathrm{O}$ & $\mathrm{MgO}$ & $\mathrm{Na}_{2} \mathrm{O}$ & $\mathrm{ZnO}$ & $\mathrm{P}_{2} \mathrm{O}_{5}$ & $\mathrm{BaO}$ & $\mathrm{ZrO}_{2}$ & $\mathrm{TiO}_{2}$ \\
VGT (TGB) & 71,2 & 2,18 & 0,10 & 9,64 & 0,02 & - & 16,8 & - & 0,02 & - & - & 0,04 \\
Lodo ETE (STP) & 58,4 & 16,2 & 0,54 & 8,06 & 2,92 & 2,32 & 1,42 & 5,11 & - & 0,82 & 4,08 & 0,13 \\
\hline
\end{tabular}


sendo classificado como resíduo perigoso segundo a Norma ABNT-NBR 10.004. Informações detalhadas sobre a preparação e caracterização do lodo de ETE podem ser obtidas em estudo anterior [9]. O vidro de garrafa transparente (VGT) de coloração clara (tipo sódico-cálcico) foi triturado em um britador de martelos (Servitech, CT-058) e o produto resultante moído por $30 \mathrm{~min}$ em moinho rápido (Servitech, CT-242) com jarro de porcelana contendo bolas de alumina e água, tal que pós com tamanhos de partículas menores que $44 \mu \mathrm{m}$ (passantes em peneira de $325 \mathrm{mesh}$ ), após moagem, foram obtidos $\left(\mathrm{d}_{50}=6 \mu \mathrm{m}\right)$. Subsequentemente, os pós obtidos foram secos $\left(110^{\circ} \mathrm{C} / 2 \mathrm{~h}\right)$ em estufa (SP Labor) e misturas (mistura a seco em moinho de bolas por $30 \mathrm{~min}$ ) contendo diferentes proporções de VGT $(50-70 \%$ em massa), de lodo de ETE (30-50\% em massa) e de carbonato de cálcio (2-10\% em massa) foram preparadas com adição de $5 \%$ de água.

As misturas dos pós foram, em uma etapa posterior, compactadas uniaxialmente em matriz de aço por meio de uma prensa hidráulica (Bovenau, P10 ST) a $40 \mathrm{MPa}$. Os corpos de prova obtidos, com $30 \mathrm{~mm}$ de diâmetro e 10 $\mathrm{mm}$ de espessura, foram secos em estufa a $110{ }^{\circ} \mathrm{C}$ por $2 \mathrm{~h}$. O comportamento térmico durante queima dos pós das matérias-primas e das misturas preparadas foi estudado por meio de análises em dilatômetro ótico (Expert System Solution, Misura ODHT) a $10{ }^{\circ} \mathrm{C} / \mathrm{min}$ (atmosfera oxidante). Com base nas análises dilatométricas, corpos de prova (30 mm x $10 \mathrm{~mm}$ ) de compactos de pós foram queimados em diferentes temperaturas $\left(750-1000^{\circ} \mathrm{C}\right)$ e tempos $(15-60 \mathrm{~min}) \mathrm{e}$ submetidos a diferentes medidas e análises.

A densidade aparente/geométrica, $\rho_{\mathrm{a}}\left(\mathrm{g} / \mathrm{cm}^{3}\right)$ foi calculada (Equação A) a partir de medidas de massa (m) em balança (Shimadzu, AX200, precisão de $\pm 0,001 \mathrm{~g}$ ) e de volume (v), por meio de um paquímetro digital (Mitotoyo, precisão de \pm $0,01 \mathrm{~mm})$, dos corpos de prova.

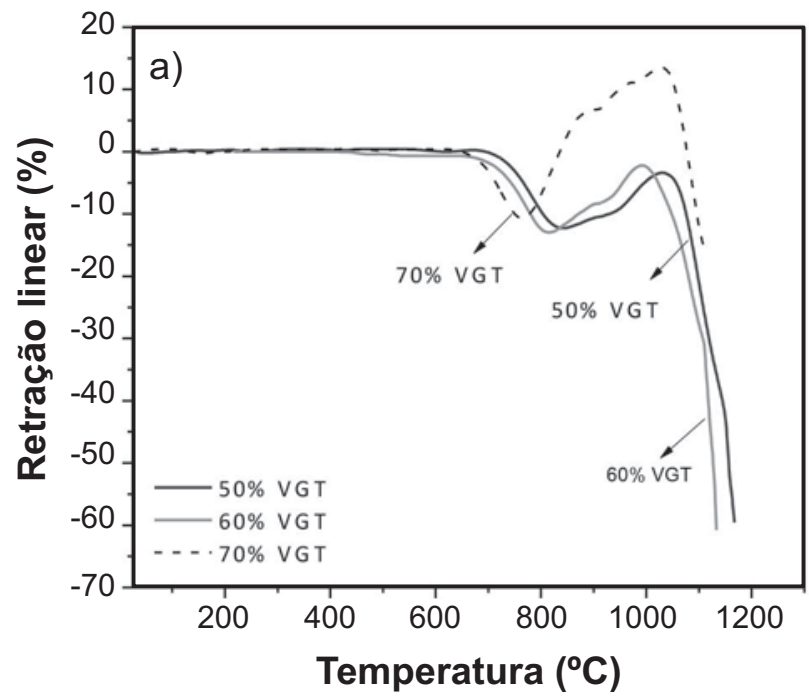

$$
\rho_{a}=m / v
$$

A densidade real, $\rho_{\mathrm{t}}\left(\mathrm{g} / \mathrm{cm}^{3}\right)$ dos pós dos materiais estudados foi determinada por picnometria a gás hélio (AccuPyc 1340, Micromeritics). A fração de vazios, porosidade $(\varepsilon)$, foi determinada considerando a relação entre as densidades (Equação B).

$$
\varepsilon=\left[1-\left(\rho_{d} / \rho_{t}\right)\right] \cdot 100
$$

A microestrutura de poros pôde ser visualizada a partir de imagens das superfícies de fraturas das amostras tratadas termicamente, obtidas em microscópio eletrônico de varredura, MEV (Philips, XL-30). Para determinar a resistência mecânica das amostras, ensaios de compressão (EMIC, DL 2000) foram realizados em 5 amostras $(30 \mathrm{~mm}$ x $10 \mathrm{~mm}$ ) com velocidade de carregamento de $1 \mathrm{~mm} / \mathrm{min}$. A condutividade térmica dos materiais obtidos foi determinada em um equipamento TCi Thermal Conductivity Analayzer, C-THERM Technologies (princípio da medida: modified transiente plane source), em amostras em forma de disco com $30 \mathrm{~mm}$ de diâmetro e $10 \mathrm{~mm}$ de espessura. As medidas foram realizadas a aproximadamente $25{ }^{\circ} \mathrm{C}$. Portanto, os valores de condutividade térmica das amostras das composições queimadas em diferentes temperaturas são indicativos do que poderiam ser os valores de condutividade térmica em temperaturas elevadas, já que neste caso os fenômenos de irradiação começam a se tornar relevantes afetando esta propriedade térmica.

\section{RESULTADOS E DISCUSSÃO}

As Figs. 1a e 1b mostram curvas de retração linear, referentes a misturas de VGT $(50-70 \%$ em massa) e de lodo de ETE (30-50\% em massa) e misturas com $70 \%$ de

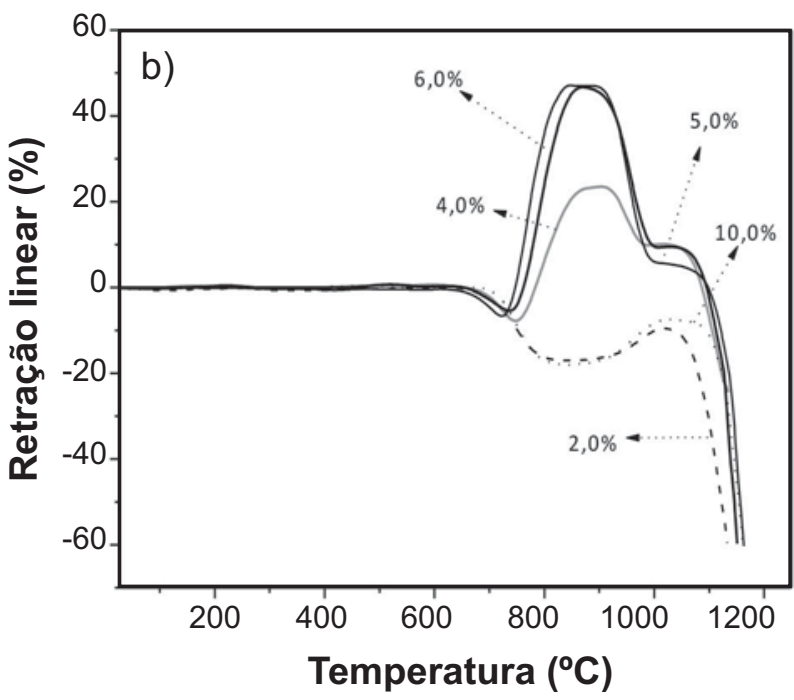

Figura 1: Curvas de retração linear referentes a misturas de (a) VGT (50-70\% em massa) e de lodo de ETE (30-50\% em massa) e (b) misturas com $70 \%$ de VGT e $30 \%$ de lodo de ETE com diferentes quantidades de $\mathrm{CaCO}_{3}(2-10 \%$ em massa).

[Figure 1: Linear shrinkage curves related to mixtures of (a) TGB (VGT) (50-70 wt\%) and sludge from sewage treatment plant, STP (30-50 $w t \%)$ and (b) mixtures with $70 \%$ TGB and $30 \%$ STP sludge with different amounts of $\left.\mathrm{CaCO}_{3}(2-10 \mathrm{wt} \%).\right]$ 
VGT e 30\% de lodo de ETE com diferentes quantidades de $\mathrm{CaCO}_{3}(2-10 \%$ em massa), respectivamente. Como pode ser observado na Fig. 1a, a expansão relacionada ao amolecimento do vidro ocorre quando a quantidade de VGT é de pelo menos $70 \%$. Observa-se também que a expansão ocorre no intervalo de temperatura compreendido entre 750 e $1050{ }^{\circ} \mathrm{C}$ (máxima expansão), o que inclui temperaturas onde se verifica a decomposição do carbonato cálcio, isto é, de 700 a $800{ }^{\circ} \mathrm{C}$. Por esta razão, os compostos com $70 \%$ VGT e 30\% de lodo de ETE foi escolhido já que é aquele que apresenta quantidades otimizadas das matérias-primas utilizadas e associadas ao comportamento desejado. De fato, conforme Fig. 1b, a expansão máxima ocorre a $850{ }^{\circ} \mathrm{C}$ e é crescente para adições de carbonato de cálcio entre 2 e $6 \%$. Para quantidades maiores de carbonato de cálcio (10\%) não ocorre expansão. Este comportamento está relacionado, provavelmente, a um aumento do volume de gases da decomposição do $\mathrm{CaCO}_{3}$, que resulta em aumento da pressão interna e assim o rompimento das paredes dos poros permitindo o escape dos gases não promovendo a expansão do corpo cerâmico. Desta maneira, a composição com 70\% de VGT e 30\% de lodo de ETE e com 5\% de carbonato de cálcio foi escolhida, pois apresenta as condições favoráveis em termos das quantidades relativas das matérias-primas utilizadas e possibilidade de obtenção de um material poroso.

A Fig. 2 mostra gráfico relacionando a porosidade em função da temperatura e do tempo de permanência na temperatura de queima de composição contendo $70 \%$ de VGT, 30\% de lodo de ETE e 5\% de carbonato de cálcio. Como pode ser visto, a porosidade aumenta $(\sim 3$ - 62\%) à medida que a temperatura de queima se eleva de

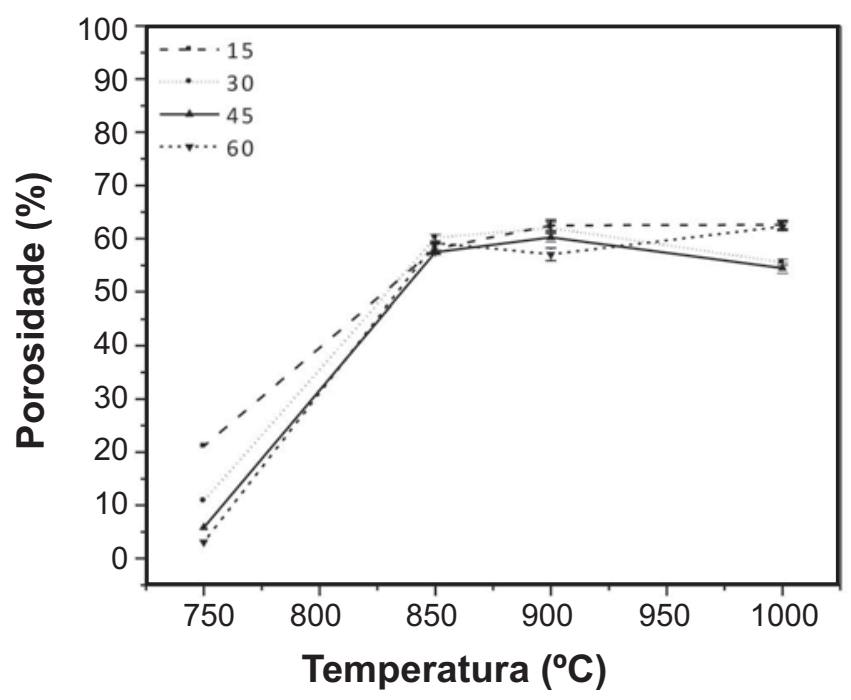

Figura 2: Gráfico relacionando a porosidade em função da temperatura e do tempo de permanência (15-60 min) na temperatura de queima de composição contendo $70 \%$ de VGT, $30 \%$ de lodo de ETE e 5\% de carbonato de cálcio.

[Figure 2: Graph relating the porosity as a function of temperature and holding time $(15-60 \mathrm{~min})$ in the firing temperature for a composition containing 70\% TGB, 30\% of sludge from sewage treatment plant, STP and 5\% of calcium carbonate.]
750 para $850{ }^{\circ} \mathrm{C}$ (temperatura de máxima porosidade), mantendo-se praticamente constante entre 850 e $1000{ }^{\circ} \mathrm{C}$, independentemente do tempo de permanência na temperatura. Todavia, na temperatura de $750^{\circ} \mathrm{C}$ ocorre uma diminuição da porosidade à medida que o tempo na temperatura de queima aumentou de 15 para $60 \mathrm{~min}$. Nesta temperatura, o corpo cerâmico atinge sua máxima densificação, como mostrado na Fig. 1 (em termos de retração), a qual se intensifica à medida que o tempo na temperatura de queima aumenta. Além disso, nesta temperatura, os gases resultantes da decomposição do carbonato de cálcio começam a agir dando início à expansão. Por esta razão é que os corpos cerâmicos queimados a $750{ }^{\circ} \mathrm{C}$ por $15 \mathrm{~min}$, por exemplo, apresentaram maiores porosidades que aqueles queimados na mesma temperatura para tempos maiores que 15 min e menores que $60 \mathrm{~min}$. De fato, as micrografias da Fig. 3 evidenciam estes aspectos mencionados. Percebe-se, inclusive, que à medida que a temperatura aumenta ocorre um aumento do tamanho (coalescimento) dos poros (Figs. 3a-3d), referentes às amostras queimadas entre 750 e $1000{ }^{\circ} \mathrm{C}$ por $60 \mathrm{~min}$, associado, ainda, a uma distribuição de tamanhos de poros mais ampla (com poros pequenos e grandes), em particular para as amostras queimadas a temperaturas maiores que $900{ }^{\circ} \mathrm{C}$ (Figs. 3c e 3d).

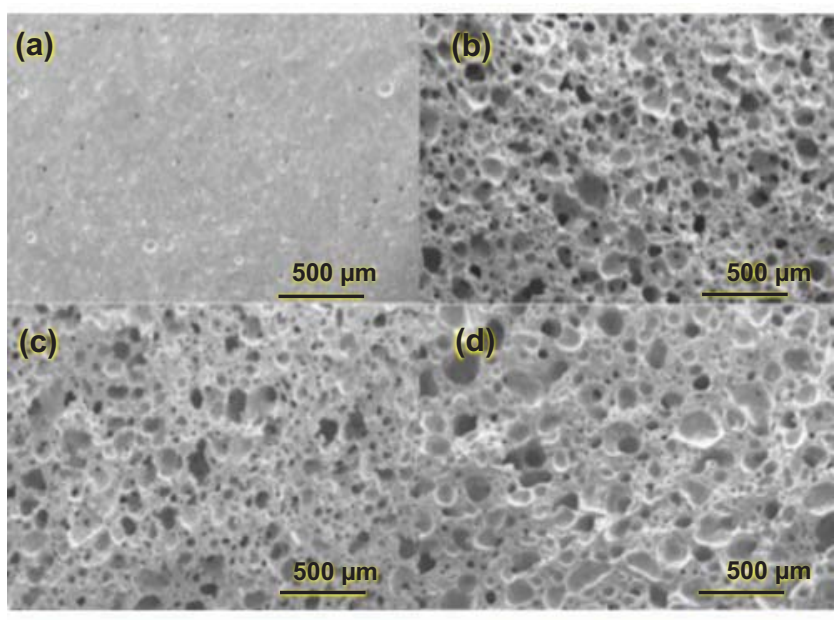

Figura 3: Micrografias (MEV) mostrando a microestrutura de poros de amostras (composição contendo $70 \%$ de VGT, 30\% de lodo de ETE e $5 \%$ de carbonato de cálcio) queimadas em diferentes temperaturas (a) $750{ }^{\circ} \mathrm{C}$, (b) $850{ }^{\circ} \mathrm{C}$, (c) $900{ }^{\circ} \mathrm{C}$ e (d) $1000{ }^{\circ} \mathrm{C}$ por $60 \mathrm{~min}$.

[Figure 3: SEM micrographs showing the microstructure of pores from samples (composition containing TGB 70\%, 30\% STP sludge and $5 \%$ calcium carbonate) fired at different temperatures (a) $750{ }^{\circ} \mathrm{C}$, (b) $850{ }^{\circ} \mathrm{C}$, (c) $900{ }^{\circ} \mathrm{C}$ and (d) $1000^{\circ} \mathrm{C}$ for $\left.60 \mathrm{~min}.\right]$

A Fig. 4 mostra o gráfico de condutividade térmica em função da temperatura de queima $\left(750\right.$ a $1000{ }^{\circ} \mathrm{C}$ por $60 \mathrm{~min}$ ) de amostras de composição contendo $70 \%$ de VGT, 30\% de lodo de ETE e 5\% de carbonato de cálcio. A condutividade térmica (Fig. 4), como esperado, diminui à medida que a porosidade aumenta. No entanto, ela diminuiu pouco $(\sim 1,25-1,15 \mathrm{~W} / \mathrm{m} . \mathrm{K})$ para amostras queimadas entre 750 e $900{ }^{\circ} \mathrm{C}$ e mais acentuadamente 


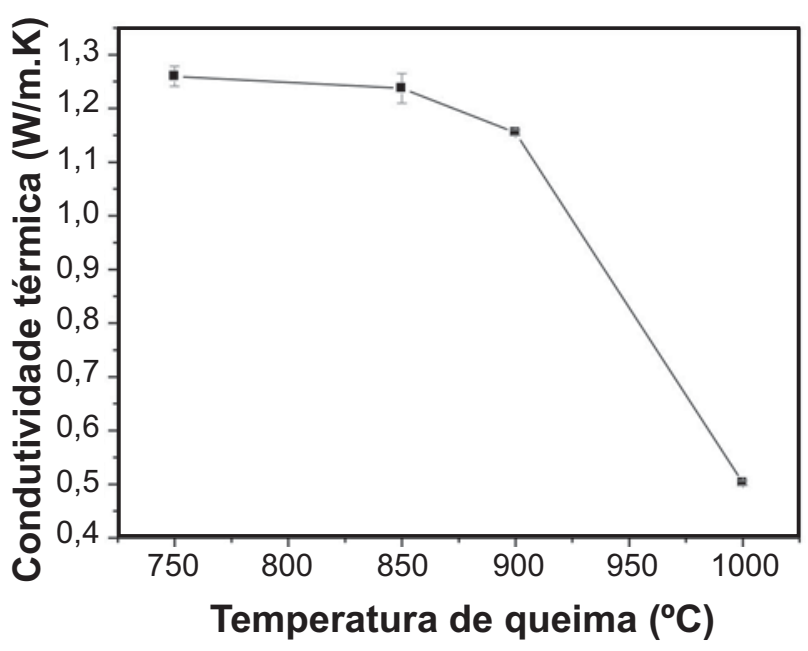

Figura 4: Gráfico de condutividade térmica em função da temperatura de queima $\left(750,850,900\right.$ e $1000{ }^{\circ} \mathrm{C}$ por $\left.60 \mathrm{~min}\right) \mathrm{de}$ amostras de composição contendo $70 \%$ de VGT, $30 \%$ de lodo de ETE e 5\% de carbonato de cálcio.

[Figure 4: Graph of thermal conductivity as a function of the firing temperature $\left(750,850,900\right.$ and $1000{ }^{\circ} \mathrm{C}$ for $60 \mathrm{~min}$ ) of samples containing $70 \%$ TGB 30\% STP sludge and $5 \%$ calcium carbonate.]

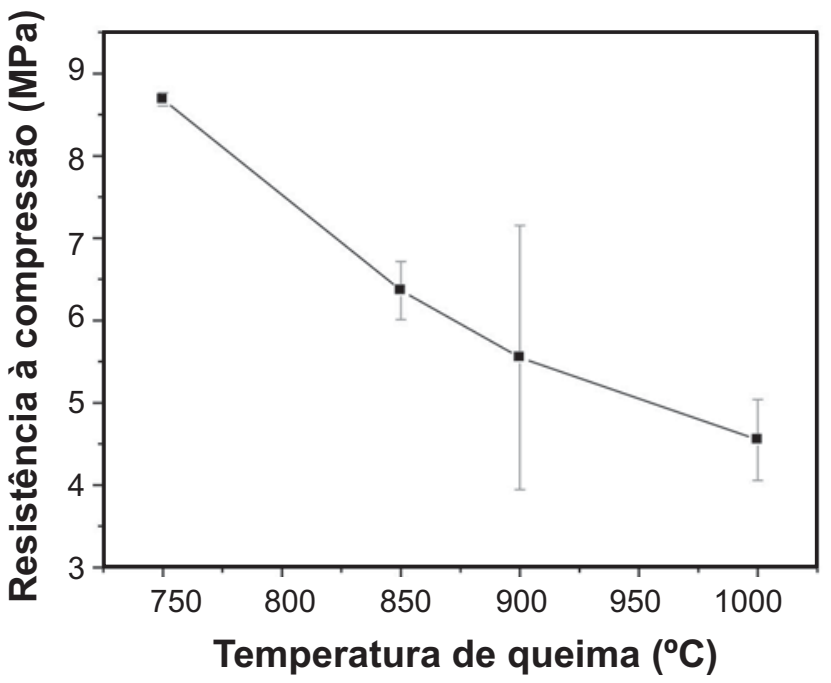

Figura 5: Gráfico de resistência à compressão em função da temperatura de queima $\left(750,850,900\right.$ e $1000{ }^{\circ} \mathrm{C}$ por $\left.60 \mathrm{~min}\right) \mathrm{de}$ amostras de composição contendo $70 \%$ de VGT, 30\% de lodo de ETE e 5\% de carbonato de cálcio.

[Figure 5: Graph of compressive strength as a function of the firing temperature (750, 850, 900 and $1000{ }^{\circ} \mathrm{C}$ for $\left.60 \mathrm{~min}\right)$ of samples containing $70 \%$ TGB, 30\% STP sludge and $5 \%$ calcium carbonate].

$(\sim 0,5 \mathrm{~W} / \mathrm{m} . \mathrm{K})$ para amostras queimadas a $1000{ }^{\circ} \mathrm{C}$ por 60 min. Tal comportamento está relacionado, provavelmente, à maior diferença de tamanhos de poros e isolamento dos mesmos nas amostras queimadas a $1000{ }^{\circ} \mathrm{C}$ por $60 \mathrm{~min}$, como evidenciado na Fig. 3d.

A Fig. 5 mostra o gráfico de resistência à compressão em função da temperatura de queima $\left(750 \mathrm{a} 1000{ }^{\circ} \mathrm{C}\right.$ por $\left.60 \mathrm{~min}\right)$ de amostras de composição contendo 70\% de VGT, 30\% de lodo de ETE e 5\% de carbonato de cálcio. A partir da análise da Fig. 5, pode-se perceber que a resistência à compressão diminui $(8,7$ a 5,4 $\mathrm{MPa})$ à medida que a temperatura de queima aumenta. Este resultado está em bom acordo com os resutados de porosidade observados. No entanto, verifica-se uma maior dispersão (desvio das medidas) em torno dos valores médios à medida que a temperatura de queima aumenta em particular para as amostras queimadas a 900 e $1000{ }^{\circ} \mathrm{C}$, o que pode ser relacionado ao aumento das dimensões dos poros associado a uma distribuição de tamanhos mais ampla. De fato, é sabido que poros maiores (maior dimensão do defeito) aumentam o fator de concetração de tensões resultando em valores de resistência mecânica menores $(5,4 \mathrm{MPa})$ para uma mesma porosidade.

\section{CONCLUSÕES}

A expansão relacionada ao amolecimento do vidro ocorre quando a quantidade de vidro (VGT) é de pelo menos $70 \%$. Observa-se também que a expansão ocorre no intervalo de temperatura compreendido entre 750 e $1050{ }^{\circ} \mathrm{C}$ (máxima expansão), o que inclui temperaturas onde se verifica a decomposição do carbonato de cálcio $\left(700\right.$ a $\left.800^{\circ} \mathrm{C}\right)$ e, assim, a geração de porosidade. Portanto, é possível produzir isolantes térmicos, a partir de composições otimizadas contendo $70 \%$ de VGT, 30\% de lodo de ETE e 5\% de $\mathrm{CaCO}_{3}$, queimados a temperaturas compreendidas entre 750 e $1000{ }^{\circ} \mathrm{C}$ por $60 \mathrm{~min}$, com porosidades entre $3 \mathrm{e}$ $62 \%$, condutividades térmicas entre 1,25 e $0,5 \mathrm{~W} / \mathrm{m} . \mathrm{K}$ e resistência à compressão entre 8,7 e 5,4 MPa. Os materiais obtidos são fortes candidatos em aplicações estruturais, tais como painéis para isolamento térmico, os quais requerem uma combinação adequada de condutividade térmica, porosidade e resistência mecânica.

\section{AGRADECIMENTOS}

Os autores agradecem a FAPESC e o CNPq (PRONEX T.O. $\left.N^{\circ} 17431 / 2011-9\right)$.

\section{REFERÊNCIAS}

[1] J.P. Holman, Transferência de Calor, McGraw-Hill do Brasil (1983) 639.

[2] J. Zeschky, F. Goetz-Neunhoeffer, J. Neubauer, S.H. Jason Lo, B. Kummer, M. Scheffler, P. Greil, Compos. Sci. Technol. 63 (2003) 2361.

[3] P. Sepulveda, J.G.P. Binner, J. Eur. Ceram. Soc. 19 (1999) 2059.

[4] F.S. Ortega, A.E.M. Paiva, J.A. Rodrigues, V.C. Pandolfelli, Cerâmica 49, 309 (2003) 1.

[5] V.R. Salvini, M.D.M. Innocentini, V.C. Pandolfelli, Cerâmica 46, 298 (2000) 97.

[6] X. Zhu, D. Jiang, S. Tan, Mater. Sci. Eng. A 323 (2002) 232.

[7] D. M. Liu, Ceram. Int. 23 (1997) 135.

[8] O.L. Alves, I.F. Gimenes, I.O. Mazalli, Cadernos Temáticos Química Nova na Escola - Novos Materiais, 
Edição Especial, fevereiro (2001) 9.

[9] V.S Nandi, J. Feltrin, M.V. Cataneo, K.A. Scremin, O.R.K. Montedo, A.P.N. Oliveira, Cerâmica Industrial 17 (2012) 32.

[10] Associação Brasileira de Normas Técnicas - ABNT, NBR 10004, "Resíduos Sólidos - Classificação", ABNT Rio de Janeiro (2004).

[11] F.R. Pereira, A.P. Nunes, A.M. Sedagães, J.A. Labrincha, Key Eng. Mater. 264 (2004) 1743.

[12] F.R. Pereira, D. Hotza, A.M. Sedagães, J.A. Labrincha, Ceram. Int. 32 (2006) 173.
[13] M.C. Casagrande, M.N. Sartor, V. Gomes, V.P. Della, D. Hotza, A.P.N. Oliveira, Cerâmica Industrial 13, 1/2 (2008) 34 .

[14] C. Martínez-Garcia, D. Eliche-Quesada, L. PérezVillarejo, F.J. Iglesias-Godino, F.A. Corpas-Iglesias, J. Environ. Manage 95 (2012) S343.

[15] V.S. Nandi, T.S. Mondo, B.G. Oliveira, O.R.K. Montedo, A.P.N. Oliveira, Cerâmica Industrial 15, 1 (2010) 34.

[16] F. Galembeck, Química Nova 36, 10 (2013)1600. (Rec. 12/03/2015, Rev. 22/05/2015, Ac. 10/10/2015) 